\title{
Dimensión de la cultura popular y tradicional como recurso para el desarrollo local
}

\section{Dimension of popular and traditional culture as a resource for local development popular culture}

\author{
MSc, Silvio Alejandro Alcívar Molina ${ }^{1}$ \\ s alcivar1@hotmail.com
}

Recibido: 1/04/2018, Aceptado: 1/06/2018

\begin{abstract}
RESUMEN
El desarrollo local como proceso histórico concreto tiene un carácter multidisciplinar. La cultura popular y tradicional es una de las dimensiones del desarrollo local que si es potenciada puede revertirse en beneficio de los individuos. Existe en el orden teórico y metodológico una escasez de fuentes de información científico-técnica que fundamenten el papel de la cultura popular y tradicional para el desarrollo local. Se plantean como objetivos del estudio: 1) examinar los principales aspectos que tipifican el desarrollo local y la cultura popular y tradicional y 2) identificar los nexos teóricos entre el desarrollo local y la cultura popular y tradicional. Se plantean los postulados teóricos presentes entre la cultura popular y tradicional y el desarrollo local. Se enuncian aspectos que contribuirán a fortalecer el desarrollo local desde la cultura popular y tradicional.
\end{abstract}

Palabras clave: cultura popular y tradicional, desarrollo local, desarrollo comunitario, dimensiones del desarrollo

\begin{abstract}
Local development as a concrete historical process has a multidisciplinary character. Popular and traditional culture is one of the dimensions of local development that if it is enhanced can be reverted to the benefit of individuals. There is in the theoretical and methodological order a shortage of sources of scientific and technical information that support the role of popular and traditional culture for local development. The objectives of the study were: 1) to examine the main aspects that typify local development and popular and traditional culture; and 2) to identify the theoretical links between local development and popular and traditional culture. The theoretical postulates present between popular and traditional culture and local development are proposed. It outlines aspects that will contribute to strengthen local development from popular and traditional culture.
\end{abstract}

\footnotetext{
${ }^{1}$ Universidad Técnica de Manabí, Ecuador
} 


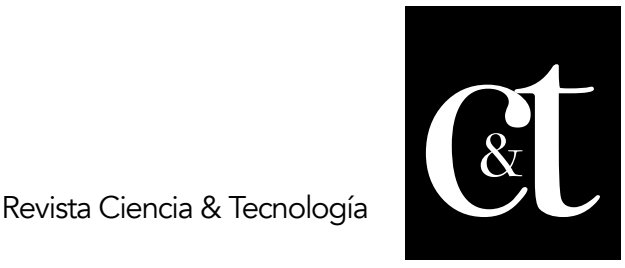

No. 19, 31 de julio de 2018

ISSN impreso: 1390 - 6321

Keywords: popular and traditional culture, local development, community development, dimensions of development

\section{Introducción}

El término de desarrollo comunitario constituye una construcción epistemológica donde comunitario constituye una cualidad. El modo la expresión fue acuñada en el contexto del "descubrimiento" de América y la certeza de que la tierra era redonda y finita. Los avances de la ciencia y la tecnología fueron la génesis del término desarrollo tal como es empleado en la actualidad. Pero el desarrollo del capital a grandes escalas supuso que se estructurara un nuevo paradigma enfocado en el movimiento ascendente de fuerzas productivas y la acumulación de capital financiero. El término progreso hacía referencia al desarrollo y servía para medir el grado de capital o industrialización que iban alcanzando unas naciones respecto a otras. La idea del progreso suponía un salto superior, sinónimo de evolución, ascenso.

La concepción sobre el desarrollo tal como se conoce en la actualidad deviene de la postguerra luego del conflicto de la segunda guerra mundial donde surge la necesidad de su conceptualización (Figueroa, 2009). Luego del enfrentamiento bélico surgen los términos que se derivan del desarrollo tales como subdesarrollo, países en vías de desarrollo, desarrollo local, desarrollo humano, desarrollo sostenible, entre otros (Boisier, 1999). Las cualidades del desarrollo se expresan "en una variedad de producciones teóricas y de prácticas donde el desarrollo se ha venido enunciando en formulaciones tales como social, humano, sostenible, multidimensional, endógeno, ecodesarrollo, autodesarrollo" (Riera, 2012). Existen otras cualidades como local, sostenible, sustentable, entre otros; y que sirven para designar los objetivos, propósitos y vías del desarrollo.

El desarrollo local como categoría refiere al espacio geográfico y aboga por un salto cualitativo de los miembros de una localidad. Lo local como categoría del desarrollo deviene en una serie de prácticas llevadas a cabo para la realización de las potencialidades de los miembros de la localidad. La cultura popular y tradicional constituye un aspecto esencial para un verdadero desarrollo local. Se expresa en las diversas manifestaciones culturales forjadas a lo largo de los años y de un alto arraigo popular.

\section{Desarrollo}

Existe en el orden teórico y metodológico insuficientes fuentes de información científico- técnica que fundamenten el papel de la cultura popular y tradicional para el desarrollo local. Se plantean como objetivos del estudio: 1) examinar los principales aspectos que tipifican el desarrollo local y la cultura popular y tradicional y 2) identificar los nexos teóricos entre el desarrollo local y la cultura popular y tradicional. El presente estudio contribuirá a identificar los principales nexos conceptuales entre la cultura popular y tradicional y el desarrollo local. La investigación aportará en el orden teórico los aspectos contenidos en el desarrollo local que pueden ser fortalecidos desde la cultura popular y tradicional.

\section{Materiales y métodos}

El estudio que se presenta clasifica como investigación descriptiva. Para la obtención de resultados se emplearon métodos en los niveles teórico y empíricos. En el nivel teórico se utilizaron los métodos analítico-sintético, sistémicoestructural, histórico-lógico e inductivo-deductivo. En el nivel empírico se 


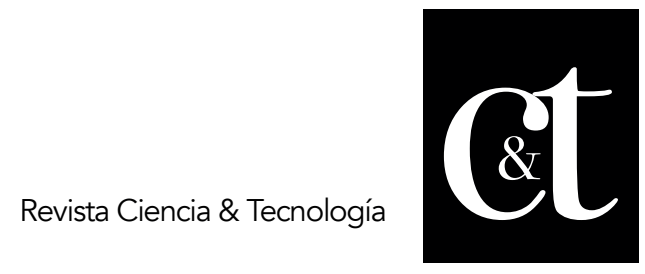

No. 19, 31 de julio de 2018

ISSN impreso: 1390 - 6321

emplearon la observación y el análisis documental clásico. Para el estudio documental realizado como forma de obtención de los referentes documentales necesarios para la realización de un estudio de tal envergadura se seleccionaron las siguientes bases de datos: Google Académico, EBSCO, Redalyc, SciELO, DOAJ y el Social Sciences Citation Index de la Web of Science. La técnica utilizada fue la revisión bibliográfica.

\section{Resultados y discusión}

Dimensión cultural del desarrollo

En la actualidad existe un conjunto de acepciones para calificar el desarrollo, entre los cuales se mencionan: autodesarrollo, desarrollo sostenible, desarrollo humano, desarrollo integral, desarrollo local y desarrollo local comunitario. La noción de cultura planteada desde el marxismo, la sitúa como toda producción material de los seres humanos. Por lo tanto, ambas categorías, desarrollo y cultura, mantienen una estrecha relación independientemente del interés contemporáneo depositado por investigadores, gobiernos y organismos internacionales. Las investigaciones de Cedeño, Cuétara y Cuétara (2016), García, et al. (2015), Troitiño (2015), Rodríguez et al. (2016), Tarazona (2017) sostienen análisis de interés para la comprensión de la dimensión cultural del desarrollo. La aparición de la cultura como objeto de interés por parte de los especialistas en desarrollo, constituye un ejemplo de la tardía comprensión de su capacidad de transformación en la realidad socioeconómica. Una visión no occidentalizada de esta relación la aportan los pueblos indígenas de Latinoamérica. El concepto de desarrollo se basa en una filosofía holística, fundamentada en los valores de reciprocidad, solidaridad, equilibrio y colectividad. Aspecto que sitúa a los seres humanos dentro de los límites del mundo natural, con fuerte arraigo a la identidad, los derechos colectivos, la seguridad y control de las tierras, los territorios y los recursos. La tradición se evidencia como elemento relevante, el respeto por los antepasados, los sistemas sociales, culturales, políticos y espirituales.

En los estudios sociológicos contemporáneos, las concepciones sobre el papel de la cultura en el desarrollo adquieren dimensiones estratégicas, pues sus contenidos se relacionan con problemáticas situadas en el centro de la discusión actual en este campo del conocimiento. Lo cultural es subestimado a partir de asociaciones univocas con el arte, la creación o expresión espiritual. Lo anterior desvincula la producción material de los procesos económicos, políticos y sociales. En ese sentido, la Declaración de las Naciones Unidas sobre los derechos de los pueblos indígenas proporciona una base legal y conceptual para afirmar sus derechos y definir sus aspiraciones con los Estados y las empresas en lo que se refiere al desarrollo con cultura e identidad (ONU, 2007).

Otras visiones alternativas al pensamiento hegemónico que marcan una tendencia a repensar el desarrollo desde perspectivas culturales la abordan autores como Hidalgo (2011). Actualmente los indicadores del desarrollo se basan en la lógica del mercado y sus consecuencias proponen una sociedad cada vez más fragmentada. El informe de desarrollo humano (PNUD, 2016) arroja datos favorables para un análisis que cualifique la dimensión cultural en este proceso. Se toman en cuenta elementos como pluralismo, cohesión social, diálogo cultural, seguridad jurídica y satisfacción de necesidades humanas básicas (libertad, creación, ocio, afecto y protección). Por tanto, se asume que la dimensión cultural contempla rasgos espirituales y materiales que caracterizan a una localidad y abarca las artes, las letras, las ciencias, las instituciones, los modos de vida, las maneras de vivir juntos, los sistemas de valores, las tradiciones y las creencias. 


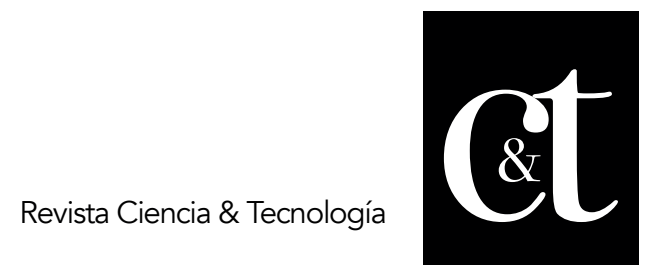

No. 19, 31 de julio de 2018

ISSN impreso: 1390 - 6321

Existe una escasa producción científica que visibilice experiencias que trasciendan el paradigma de un desarrollo economicista. En relación con esta problemática abordada anteriormente los autores Valarezo y Torres (2007) establecen que: "seguimos subestimando la comunicación, sin entender que es una seña cultural que nos permite ser ahora y mañana". Los conocimientos de la sociología, antropología, estudios culturales, comunicación y gobernabilidad constituyen el basamento epistemológico para construir una explicación de la realidad entendida como un sistema social. La fragmentación de las ciencias en la transforma el análisis global de los fenómenos de la sociedad en ensayos de laboratorio, donde estos transcurren en estado ideal.

Una visión más consciente del desarrollo debe incorporar la construcción colectiva de un proyecto que reformule políticas públicas a favor de democratizar la cultura. Entender los procesos culturales desde su globalidad o desde su génesis local, conlleva asumir la cultura como recurso indisoluble del desarrollo. La economía cultural y el turismo cultural hacen parte de la lógica del capitalismo contemporáneo. La cultura debiera entenderse no como recurso, sino de forma holística, que transforme y evolucione a partir de las múltiples interconexiones que ha logrado la sociedad (Yúdice, 2015).

La cultura es un concepto social, es dinámica y cambiante, y es "importante comprender quien está intentando atrapar (y decodificar) la cultura y con qué fines..." (Goodman, 2007). Por cuanto se presenta una antítesis entre la cultura como derecho frente a cultura como recurso. El autor Rowan (2016) critica la política cultural de España, en franca propuesta economicista que no promueve una cultura popular y democrática sino inmersa en la enajenación capitalista. En este contexto mercantil, resulta generalmente un déficit garantizar altos niveles de acceso a la ciudadanía, preservar el patrimonio y acervo cultural.

La Agenda 21 constituye otra perspectiva de análisis sobre la cultura como reto del desarrollo. Un balance de sus postulados evidencia la esencia hegemónica de sus creadores, pero plantea la necesidad de contribuir al desarrollo cultural a nivel local, nacional y global, al creer "firmemente, que la cultura puede ayudar en la lucha contra la pobreza y en el fortalecimiento de las comunidades" (Burgos, 2016). En el documento programático se asumen como evidencia internacional reunida, el papel de la cultura en el desarrollo y el sesgo de proyectos de desarrollo que no han considerado la dimensión cultural. También de la fuente mencionada se asume la garantía de la cultura para el bienestar colectivo.

La cultura como fin, constituye un motor para la diversidad, la auto realización personal y colectiva, estímulo para la creatividad e innovación. Visiones polarizadas del desarrollo, limitan la dimensión de la cultura al desvincularla de su tratamiento como recurso. Representan una base de emprendimiento las actividades, bienes y servicios culturales, vinculadas con el patrimonio, las identidades, los significados y los valores. El sentido holístico de estos elementos es funcional desde una visión comunitaria y su entendimiento solo es posible desde la contradicción como fuente generadora de desarrollo.

Cultura, desarrollo y desarrollo local

Dentro de las dimensiones de la cultura aparece la episteme desarrollo. Los estudios sociológicos sitúan en el debate contemporáneo lo relacionado con la cultura y sus dimensiones, entre ellas el constituir un recurso para el desarrollo local. 


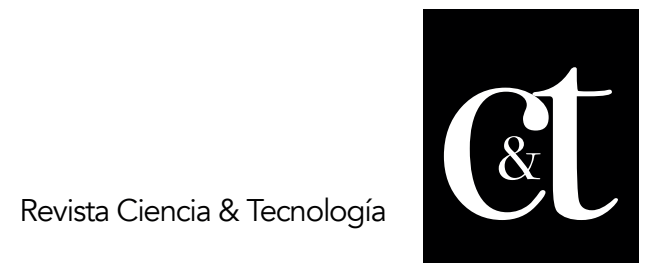

No. 19, 31 de julio de 2018

ISSN impreso: 1390 - 6321

Desde la segunda mitad de los ochenta del pasado siglo, se desarrolló el concepto de desarrollo sostenible en tres dimensiones, como pautas para las estrategias de desarrollo regional y local: crecimiento económico, inclusión social y equilibrio medioambiental. El desarrollo sostenible incluye a la cultura como componente esencial de estos procesos, al considerarse su cuarto pilar aspecto que se enfatiza en la Agenda 21 para la cultura (Weng, 2016).

La cultura como un indicador del desarrollo, contempla la participación y el involucramiento desde la diversidad. La dimensión cultural contempla rasgos espirituales y materiales que caracterizan a una localidad y abarca las artes, las letras, las ciencias, las instituciones, los modos de vida, las maneras de vivir juntos, los sistemas de valores, las tradiciones y las creencias. Está sustentada en la búsqueda y en develar las capacidades humanas que coexisten en un determinado escenario y distinguen la creación cultural de los actores locales (Álvarez, 2008).

Esta nueva perspectiva apunta a la relación entre cultura y desarrollo a través de una dualidad: desarrollando los sectores culturales propios (patrimonio, creatividad, industrias culturales, arte, turismo cultural) y abogando para que la cultura sea debidamente reconocida en todas las políticas públicas. Las políticas públicas que desde este aspecto se relacionan con la cultura son las de economía, ciencia, comunicación, medio ambiente, cohesión social y cooperación internacional. Políticas públicas son el diseño de un marco regulatorio para la ejecución estructurada para atender un grupo de necesidades Gomes y Chaves (2016), Henao et al. (2016) y Marcon et al. (2016).

La necesidad de adquirir bienes y servicios de diversa índole, hacen que las personas estructuren sus esfuerzos para alcanzarlo. La legitimación social de las demandas parte de las necesidades particulares de los individuos. Estas pasan a convertirse en los de grandes grupos de personas, aunque no tenga reconocimiento desde las posiciones de poder que son las que a menudo trazan las políticas públicas. Los esfuerzos encaminados a la solución de demandas sociales no legitimadas desde el gobierno, debe contener una amplia participación activa de los involucrados en los esfuerzos para satisfacerlas. Si solo se consideran demandas sociales aquellas a las que el gobierno apoya o legitima, solo se obtendrán los resultados del asistencialismo que puede ser solucionado con acción gubernamental.

En relación a la concepción sobre el desarrollo tal como se conoce en la actualidad deviene de la postguerra luego del conflicto de la segunda guerra mundial donde surge la necesidad de su conceptualización (Figueroa, 2009). Luego del enfrentamiento bélico surgen los términos que se derivan del desarrollo tales como subdesarrollo, países en vías de desarrollo, desarrollo local, desarrollo humano, desarrollo sostenible, entre otros (Boisier, 1999). Las cualidades del desarrollo se expresan "en una variedad de producciones teóricas y de prácticas donde el desarrollo se ha venido enunciando en formulaciones tales como social, humano, sostenible, multidimensional, endógeno, ecodesarrollo, autodesarrollo" (Riera, 2012). Existen otras cualidades como local, sostenible, sustentable, entre otros; y que sirven para designar los objetivos, propósitos y vías del desarrollo.

La dimensión universal del desarrollo ubica los valores como punto de partida y entre se sitúa la cultura como subsistema vital. En esta dimensión el individuo construye su identidad y mediante una comunidad de múltiples códigos culturales, configura sus modos de hacer y de pensar (lenguaje, costumbres, tradiciones, 


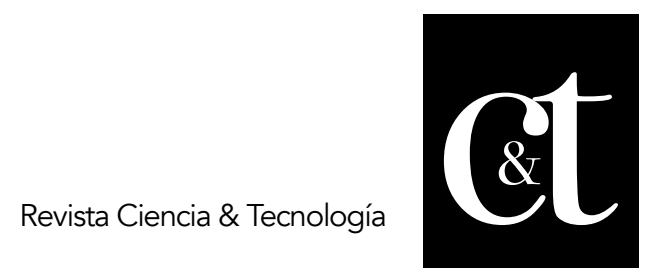

No. 19, 31 de julio de 2018

ISSN impreso: 1390 - 6321

modos de hacer, interacción grupal y familiar, sistema de creencias, procederes mágico-religiosos, entre otros) (Alcívar, Martínez, Alcívar y Paz, 2017).

La concepción sociológica de cultura repercute en la apreciación del presente y tiene una proyección hacia el desarrollo. Diversas instituciones internacionales y autores reconocen variadas dimensiones del desarrollo local, entre ellos la Unesco (2002) en la Declaración sobre el derecho al desarrollo. En el documento mencionado se define al desarrollo como "un proceso económico, social, cultural y político para el bienestar de la población, con la participación activa, libre y significativa en la distribución justa de los beneficios que de él se derivan donde la persona humana es el sujeto central del proceso" (ONU, 2017). El desarrollo local es un proceso de carácter objetivo. Es multicondicionado y multidireccional. Algunas de las variables e indicadores relacionadas con el desarrollo local son eficiencia, equidad, sostenibilidad, cooperación, seguridad, potenciación y participación.

Otra dimensión del desarrollo local es considerada como desarrollo endógeno, el cual plantea como cualidades:

- No ser únicamente desarrollo de la localidad, es decir, el territorio como sistema económico social está compuesto por un complejo y contradictorio entramado de relaciones sociales que se dan entre los agentes en un contexto histórico determinado.

- No solo ser desarrollo endógeno: muchas iniciativas se basan en oportunidades del dinamismo exógeno.

- El desarrollo local es un enfoque territorial de «abajo-arriba»; precisa de una estrecha coordinación entre los diferentes niveles.

- No se limita al desarrollo económico-social: es un enfoque integrado donde intervienen elementos históricos, sociales, culturales, ambientales, institucionales y de desarrollo humano.

Entre los núcleos esenciales del desarrollo endógeno se consideran:

- Aspecto geo-histórico: existencia de un acervo cultural y tradiciones en la comunidad

- Base socioeconómica: distinción de la estructura según el tipo de economía con base en las formas de propiedad sobre los medios de producción

- Capacidad productiva y tecnológica

- Medioambiente y biodiversidad

- Cooperación entre los eslabones de la cadena productiva

- Aspecto Social: capital humano, servicios públicos, deporte y recreación

- Aspectos culturales: tradiciones, festividades populares, reforzar la identidad local, regional y nacional)

- Político-institucional: existencia del gobierno local, democracia participativa y control social, cooperativas y sindicatos, redes sociales y círculos comunitarios. Se trata, por tanto, de nivel de desarrollo que toma como unidad de actuación principal, el territorio (ámbito de una determinada comunidad local o comarcal en sus relaciones sociales y de producción) (Galindo y Hernández, 2013).

Como resumen de este proceso Fajardo y Figueras (2013) afirman que la gestión municipal para el desarrollo local, concebida desde la estrategia de desarrollo territorial, debe plantearse las siguientes tareas para lograr dicho desarrollo:

1. Orientar a los gobiernos locales hacia la captación, evaluación y solución del 
sistema de contradicciones existentes en el territorio en cuanto a producción, distribución y redistribución de los recursos.

2. Promover relaciones de cooperación en las diversas ramas de la estructura social del territorio con el fin de mejorar la articulación y diversificación productiva local.

3. Propiciar la formación de individuos de individuos capaces de ejecutar estas acciones en los territorios; enriquecimiento del capital humano y capacitación de la gestión en sus diversas esferas.

El protagonismo de los actores sociales constituye el soporte para las acciones de desarrollo local que tienen en su base la convivencia en el territorio desde la cotidianidad. El proceso debe desarrollarse desde espacios de reflexión de los actores sociales en todos sus niveles. Para encausar el desarrollo de un territorio se propone la ejecución de un proceso de planificación que siga los siguientes parámetros identificados por Fajardo y Figueras (2013):

- Recogida de información

- Análisis y diagnóstico

- Definición de la estrategia de desarrollo local: objetivos generales y líneas de actuación

- Diseño de un plan de acción e identificación de proyectos de desarrollo local

- Gestión de proyectos y ejecución de los seleccionados

A criterio de Mederos (2013) el desarrollo local se asume desde las siguientes perspectivas:

Fortalecimiento de las estructuras y poderes locales.

- Estimulación de la participación ciudadana cooperación para la interacción social

- Promoción de acciones integradas en los procesos de producción y reproducción de la vida cotidiana en la dimensión local

- Consideración del trabajo comunitario como instrumento básico de los procesos de desarrollo local

- Rescate de la identidad sociocultural

- Promover actividades de desarrollo desde lo local (municipio, barrio, asentamientos humanos)

En consideración se debe tener en cuenta las políticas públicas locales, regionales y nacionales en los que se dan los procesos de desarrollo. El reconocimiento del sistema de contradicciones que generan los diversos tipos de propiedad. Fortalecer el trabajo comunitario. Actividades productivas en lo social, cultural y ambiental. Acciones de capacitación a partir de las necesidades locales. Diversidad de los componentes de la localidad.

Los presupuestos mencionados conducen a la formulación de principios para incentivar el desarrollo local desde los gobiernos (Fajardo y Figueras, 2013):

1. Despliegue de acciones desde el entramado institucional en función de los objetivos de la Estrategia.

2. Impulsar proyectos emergidos desde las instituciones radicadas en el territorio 


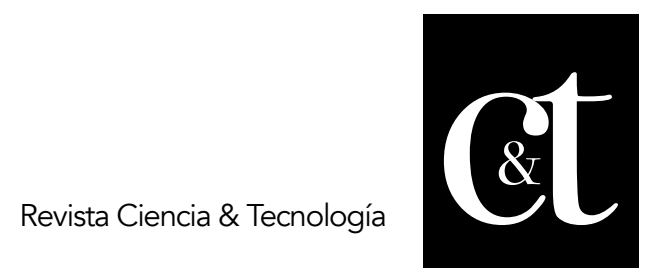

No. 19, 31 de julio de 2018

ISSN impreso: 1390 - 6321

orientadas a erradicar conflictos existentes.

3. Participación real de los actores locales en el proceso de concepción y elaboración de la Estrategia.

4. Cooperación, implicación y potenciación de todos los actores de la sociedad territorial en las acciones orientadas al desarrollo local.

5. Correspondencia entre las actividades colectivas orientadas al progreso local y las necesidades reales.

El desarrollo local y la cultura popular y tradicional deben ser dos aspectos que deben integrarse holísticamente. Debe tomarse en cualquier proyecto de desarrollo a la cultura y las tradiciones de las comunidades en los que se realicen. Las potencialidades de los individuos solo pueden alcanzar mayor grado de desarrollo si se toman en consideración las particularidades de su conformación como localidad y comunidad.

\section{Conclusiones}

Los niveles o calificaciones del desarrollo constituyen una visión fragmentada de un proceso social que se genera de acuerdo a las condiciones de los sujetos que lo gestan. La cultura como fin, constituye un motor para la diversidad, la auto realización personal y colectiva, estímulo para la creatividad e innovación.

Lo local como dimensión del desarrollo refiere a la territorialidad. En los procesos de desarrollo local intervienen varias dimensiones donde una de ella es la cultura popular y tradicional. Los procesos de desarrollo local que se ejecutan desde las tradiciones y la cultura, se repercuten en beneficio de los individuos los cuales se integran y tienen mayor identidad con los proyectos de desarrollo.

\section{Referencias bibliográficas}

Alcívar, S., Martínez, M., Alcívar, M.A. y Paz, L.E. (2017). La cultura popular tradicional en ecuador y la comunicación educativa desde las televisiones comunitarias. Revista Cognosis, 2(1), 73-82.

Álvarez, F. L. (2008). La dimensión cultural del desarrollo local. Una experiencia en el oriente cubano. Tesis de doctorado en Ciencias Sociológicas, Cuba: Universidad de Oriente.

Boisier, S. (1999). Desarrollo (local): ¿De qué estamos hablando? Recuperado de: http://www.cedet.edu.ar/sitio/administracion/agenda/boisier.pdf

Burgos, A. (2016). Buen vivir con la naturaleza en las instituciones educativas: una necesidad en Boyacá, Colombia. Culturales, 4(2), 185-208. 


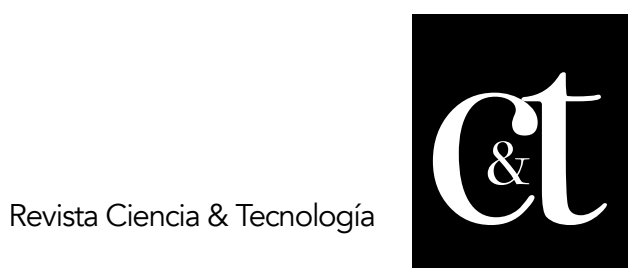

No. 19, 31 de julio de 2018

ISSN impreso: 1390 - 6321

Cedeño, T.E., Cuétara, Y., y Cuétara, L.M. (2016). Turismo cultural. Evaluación de la dimensión cultural en la gestión de la oferta turística en destinos de sol y playa. Retos Turísticos, 15(1), 1-12.

Fajardo, L y Figueras, D. (2013). Algunas consideraciones acerca de la concepción del desarrollo local. Desarrollo local y territorial. Proyectos de integración. Sancti Spíritus: Ediciones Luminaria.

Figueroa, G. (2009). Ciencias sociales cubanas del siglo veintiuno y el problema del desarrollo. Una fascinación contradictoria. Tesis presentada en opción al Título de Máster en Desarrollo Social, Cuba: Universidad de La Habana.

Galindo, S. y Hernández, M. (2013). Los valores y la cultura como subsistemas locales para la promoción del desarrollo. Desarrollo local y territorial. Proyectos de integración. Sancti Spíritus: Ediciones Luminaria.

García, C. Y., Herrera, C. I., García, C. M., y Guevara, C. E. (2015). El trabajo colaborativo y su influencia en el desarrollo de la cultura profesional docente. Gaceta Médica Espirituana, 17(1), 85-91.

Gomes, J., y Chaves, D. (2016). Trabalho e movimentos sociais: diálogo com as políticas públicas no Brasil - o caso ConCidades (2013-2014). Análise Social, 219(2), 336-365.

Goodman, D. (2007). Narrativas del cambio: Cultura y desarrollo económico regional. Confines de relaciones internacionales y ciencia política, 3(5), 19-34.

Henao, S., Quintero, S., Echeverri, J., Hernández, J., y Rivera, E. (2016). Políticas públicas vigentes de salud mental en Suramérica: un estado del arte. Revista Facultad Nacional de Salud Pública, 34(2), 184-192.

Hidalgo, A.L. (2011). Economía Política del Desarrollo. La construcción retrospectiva de una especialidad académica". Revista de Economía Mundial, No 28: 279-320.

Marcon, R., Marques, J. R., y Amaral, K. G. (2016). Participação popular e políticas públicas: uma revisão integrativa. Brazilian Journal of Surgery and Clinical Research, 16(1), 84-91.

Mederos, A. (2013). El tratamiento de las desigualdades sociales en los proyectos de desarrollo local en Villa Clara. Desarrollo local y territorial. Proyectos de integración. Sancti Spíritus: Ediciones Luminaria.

ONU (2007). Declaración de las Naciones Unidas sobre los derechos de los pueblos indígenas. Recuperado de: http://www.un.org/esa/socdev/unpfii/documents/DRIPS_es.pdf

ONU (2017). Declaración sobre el derecho al desarrollo. Recuperado de: http://www.un.org/es/events/righttodevelopment/declaration.shtml

PNUD (2016). Informe sobre Desarrollo Humano 2016. Nueva York.

Riera, C. M. (2012). Lo comunitario como cualidad del desarrollo local su alcance práctico para la transformación emancipadora de la sociedad. Tesis presentada en opción al grado científico de Doctor en Ciencias Sociológicas, Cuba: Universidad Central "Marta Abreu" de Las Villas. 
Rodríguez, N. A., Pérez, O. L., Canalda, M. E., y Ivanovna, S. (2016). Intervención comunitaria en el contexto universitario para elevar el desarrollo cultural de las familias. Ra Ximhai, 12(5), 145-166.

Rowan, J. (2016). Una economía cultural de la cultura. Periférica Internacional, $17(2016), 10-23$.

Tarazona, V. R. (2017). ¿La universidad para profesionalizar o para el desarrollo cultural? Revista Sophia, 13(1), 1-3.

Troitiño, L. (2015). La dimensión turística del patrimonio cultural de la ciudad de Lorca (Murcia, España). Cuadernos De Turismo, 36(2015), 389-414.

Valarezo, R., y Torres, V. H. (2007). El desarrollo local en el Ecuador. Historia, Actores y Métodos. Quito: Ediciones Abya-Yala.

Weng, S. S. (2016). Asset mapping for an Asian American community: Informal and formal resources for community building. Psychosocial Intervention, 25(2016), 55-62.

Yúdice, G. (2015). Internet en manos de sectores privados hoy está disputando el poder a los Estados. Recuperado de: http://www.lanacion.com.ar/1792906internet-hoy-esta- disputando-el-poder-alos-estados 